\title{
HIGH ORDER DIVERGENCE-FORM ELLIPTIC OPERATORS ON LIE GROUPS
}

\author{
A.F.M. TER Elst and Derek W. Robinson
}

We give a straightforward proof that divergence-form elliptic operators of order $m$ on a $d$-dimensional Lie group with $m \geqslant d$ have Hölder continuous kernels satisfying Gaussian bounds.

\section{INTRODUCTION}

Consider the operator

$$
H=\sum_{|\alpha|,|\beta| \leqslant n}\left(A^{\alpha}\right)^{*} c_{\alpha, \beta} A^{\beta}
$$

of order $m=2 n$ acting on $L_{2}=L_{2}(G ; d g)$ where $G$ is a $d$-dimensional Lie group with left Haar measure $d g$, the $c_{\alpha, \beta}$ are operators of multiplication by $L_{\infty}$-functions, the $A^{\alpha}, A^{\beta}$ are products of the skew-adjoint generators $A_{i}=d L\left(a_{i}\right), i \in\{1, \ldots, d\}$ of left translations on $L_{2}$ corresponding to the multi-indices $\alpha, \beta$ and $a_{1}, \ldots, a_{d}$ is a vector space basis of the Lie algebra $g$ of $G$. (Definitions and background information can be found in [17].) We assume

$$
\operatorname{Re} \sum_{|\alpha|=|\beta|=n}\left(\psi_{\alpha}, c_{\alpha, \beta} \psi_{\beta}\right) \geqslant \mu \sum_{|\alpha|=n}\left\|\psi_{\alpha}\right\|_{2}^{2}
$$

for some $\mu>0$, uniformly for all $\psi_{\alpha} \in L_{2}$, and define the ellipticity constant $\mu_{C}$ as the least upper bound of the $\mu$ for which this condition is satisfied. Then $H$ can be precisely defined as the closed maximal accretive operators associated with the sectorial forms

$$
h(\psi, \varphi)=\sum_{|\alpha|,|\beta| \leqslant n}\left(A^{\alpha} \psi, c_{\alpha, \beta} A^{\beta} \varphi\right)
$$

Received 16th September, 1996.

Parts of this work were carried out whilst the second author was visiting the Technical University in Eindhoven and the first author was visiting the Centre for Mathematics and its Applications at the ANU. Both authors wish to thank the respective institutes for supporting these visits.

Copyright Clearance Centre, Inc. Serial-fee code: 0004-9729/97 \$A2.00+0.00. 
with domain $D(h)=L_{2 ; n}$, the functions which are $n$-times differentiable in the $L_{2}$ sense. It follows that each such $H$ generates a strongly continuous, holomorphic, semigroup $S$ on $L_{2}$ (see, for example, [14, Chapter VI]) with a kernel $K_{t}$ defined as a distribution such that

$$
\left(\psi, S_{t} \varphi\right)=\int_{G} d g \overline{\psi(g)} \int_{G} \widehat{d h} K_{t}(g ; h) \varphi(h)
$$

for all $\varphi, \psi \in C_{c}^{\infty}(G)$ and $t>0$ where $d \hat{h}$ denotes right Haar measure. Our aim is to give an elementary analysis of $K_{t}$ when the dimension $d$ is small relative to $m$. Specifically we assume $d \leqslant m$ and establish that $K$ satisfies Gaussian bounds and is Hölder continuous with an order related to the relative size of $d$ and $m$.

If $G=\mathbf{R}^{d}$ and $n=1$ then $H$ corresponds to a second-order strongly elliptic partial differential operator in divergence form. Such operators are of fundamental importance in the calculus of variations and have been studied in enormous detail. It is not difficult to establish for $d=1$ that the kernels are bounded and Hölder continuous for all $t>0$. A similar result for $d=2$ follows from the early work of Morrey [15]. If $d \geqslant 3>m$, then the situation is more complicated and it is useful to distinguish two distinct cases, real coefficients and complex coefficients. For real coefficients the Hölder continuity of the kernel is a famous result established independently by De Giorgi [13] and Nash [16]. But for complex coefficients, or for systems with real coefficients, this property no longer holds if $d \geqslant 5$. The situation for $d \in\{3,4\}$ is unclear. (A discussion of counterexamples for systems, with references, is given in [12], [2] and [8].) Recently, Davies [7] has examined higher-order operators and has obtained Gaussian bounds whenever $d<m$. Our proofs are rather different and are an extension of the perturbation theory and embedding arguments given in [3, Section 3].

All subsequent estimates are in terms of the ellipticity constant $\mu_{C}$ and the norms of the coefficients. Let $M_{C}=\sup \left\{\left\|c_{\alpha, \beta}\right\|_{\infty}:|\alpha|,|\beta| \leqslant n\right\}$ where $\|\cdot\|_{\infty}$ denotes the $L_{\infty}$-norm and let $\mathcal{E}_{m}(\mu, M)$ denote the set of $H$ for which $\mu_{C} \geqslant \mu$ and $M_{C} \leqslant M$. Moreover, for $g \in G$ let $|g|$ be the Riemannian distance from $g$ to the identity element of $G$, canonically associated with the vector spaces $a_{1}, \ldots, a_{d}$. Finally the smoothness of the kernels as functions over $G \times G$ is expressed in terms of the left derivatives $A_{i}$, and $B_{i}$, with respect to the first and second variable of the kernel, respectively.

Theorem 1.1. Assume $d \leqslant m$. For each $M \geqslant \mu>0$ there is a $\nu \in\langle 0,1\rangle$ such that for all $\alpha, \beta$ and $\sigma \in\langle 0,1\rangle$ with $|\alpha|+\sigma \leqslant 2^{-1}(m-d)+\nu$ and $|\beta|+\sigma \leqslant$ $2^{-1}(m-d)+\nu$ and all $\kappa>0$ there exist $a, b>0$ and $\omega \geqslant 0$ such that for all $H \in \mathcal{E}_{m}(\mu, M)$ the corresponding kernels $K$ satisfy

$$
\left|\left(A^{\alpha} B^{\beta} K_{t}\right)(g ; h)\right| \leqslant a t^{-(d+|\alpha|+|\beta|) / m} e^{\omega t} e^{-b\left(\left|g h^{-1}\right|^{m} t^{-1}\right)^{1 /(m-1)}}
$$


and

$$
\begin{aligned}
& \left|\left(A^{\alpha} B^{\beta} K_{t}\right)\left(k^{-1} g ; l^{-1} h\right)-\left(A^{\alpha} B^{\beta} K_{t}\right)(g ; h)\right| \\
& \quad \leqslant a t^{-(d+|\alpha|+|\beta|) / m} e^{\omega t}\left(\frac{|k|+|l|}{t^{1 / m}+\left|g h^{-1}\right|}\right)^{\sigma} e^{-b\left(\left|g h^{-1}\right|^{m} t^{-1}\right)^{1 /(m-1)}}
\end{aligned}
$$

uniformly for all $g, h, k, l \in G$ and $t>0$ with $|k|+|l| \leqslant \kappa t^{1 / m}+2^{-1}\left|g h^{-1}\right|$.

In particular if $\boldsymbol{d}=m$ the kernel is Hölder continuous and satisfies Gaussian bounds but it is not necessarily differentiable. The degree of smoothness is an increasing function of the difference between $m$ and $d$. The degree of regularity is, however, not optimal. For example, if $d=1=n$ then the Hölder continuity bounds are valid for $\sigma \in\langle 0,1]$, and $|\alpha|=0=|\beta|(\operatorname{see}[\mathbf{3}, 11])$.

Note that if $H \in \mathcal{E}_{m}(\mu, M)$ and $|\theta|<\arctan (\mu / M)$ then $e^{i \theta} H \in \mathcal{E}_{m}\left(\mu^{\theta}, M\right)$ with $\mu^{\theta}=\mu \cos \theta-M|\sin \theta|$. Therefore the kernel $K_{z}$ of $S_{z}$ satisfies bounds similar to the above, with $t$ replaced by $|z|$, uniformly for $z \in \mathbf{C} \backslash\{0\}$ with $|\arg z| \leqslant \theta$.

\section{Proofs}

The proof of Theorem 1.1 is based on a combination of perturbation arguments and Sobolev embeddings which extends ideas of [3] but avoids any form of scaling. Since the proof for non-unimodular $G$ introduces some extraneous difficulties we first examine unimodular $G$ and subsequently explain the extension to non-unimodular groups. The proof for unimodular $G$ is in two steps. The first step establishes uniform bounds. The second step extends the uniform bounds to Gaussian bounds by Davies' exponential perturbation method [7].

STEP 1. (Uniform bounds: unimodular $G$ ) The proof starts with the $L_{2}$-estimates

$$
\left\|S_{t}\right\|_{2 \rightarrow 2} \leqslant e^{\omega t}, \quad\left\|H S_{t}\right\|_{2 \rightarrow 2} \leqslant a t^{-1} e^{\omega t}
$$

which yield bounds

$$
\left\|A^{\alpha} S_{t}\right\|_{2 \rightarrow 2} \leqslant a t^{-|\alpha| / m} e^{\omega t},
$$

whenever $|\alpha| \leqslant n$, for all $t>0$. (Here and in the sequel we use $a$ and $\omega$ to denote parameters whose values may vary line by line. The $a$ are strictly positive, the $\omega$ are non-negative and they are all decreasing functions of $\mu_{C}$ and increasing functions of $M_{C}$.) These estimates follow from the ellipticity condition (1) applied to $e^{i \theta} H$, the Cauchy integral formula and $\varepsilon, \varepsilon^{-1}$ arguments by standard reasoning. (Further details are given in the derivation of (15).) 
Next, since $d \leqslant m$, one has the Sobolev inequalities

$$
\left\|A^{\alpha} \varphi\right\|_{p} \leqslant \varepsilon^{n-|\alpha|-d_{\iota}} \sup _{|\beta|=n}\left\|A^{\beta} \varphi\right\|_{2}+c \varepsilon^{-|\alpha|-d \propto}\|\varphi\|_{2}
$$

with $s=1 / 2-1 / p$ for all $\varepsilon \in\langle 0,1]$ and all $\alpha$ with $|\alpha|\langle n$ whenever $s \in[0,1 / d\rangle$ (see [17, Appendix B.2]). Thus if $d=1$, or $d=2$, the inequalities are valid for all $p \in[2, \infty)$ and if $d \geqslant 3$ they are valid for $p \in[2,2 d /(d-2)\rangle$. Therefore, replacing $\varphi$ by $S_{t} \varphi$, setting $\varepsilon=t^{1 / m}$ and using the foregoing $L_{2}$-bounds, one finds

$$
\left\|A^{\alpha} S_{t}\right\|_{2 \rightarrow p} \leqslant a t^{-d s / m} t^{-|\alpha| / m} e^{\omega t}
$$

for all $\alpha$ with $|\alpha|<n$ and all $t>0$. (Initially these estimates are valid for $t \in\langle 0,1]$ and then, by use of the semigroup property, for all $t>8$.) The main technical difficulty in this first step is to establish that the bounds (5) remain valid for $|\alpha|=n$ and some $p>2$.

The proof uses the positive self-adjoint operator $\Delta_{m}$ determined by the quadratic form

$$
\varphi \mapsto \delta_{m}(\varphi)=\sum_{|\alpha|=n}\left\|A^{\alpha} \varphi\right\|_{2}^{2}
$$

with domain $L_{2 ; n}$. Then $\Delta_{m}$ is a strongly elliptic operator with constant coefficients which generates a continuous semigroup $T$ with a smooth Gaussian kernel (see [17, Chapters 1 and 3], or, for a short proof, see [10]). Consequently, for each $\alpha$ one has bounds

$$
\left\|A^{\alpha} T_{t}\right\|_{p \rightarrow q} \leqslant a t^{-|\alpha| / m} t^{-d s / m} e^{\omega t}
$$

uniformly for all $t>0$ and all $p, q \in[1, \infty]$ with $q \geqslant p$, where $s=1 / p-1 / q$. Hence if $\lambda_{0}=\omega+1,|\alpha|<n$ and $p, q \in[1, \infty]$, with $n-|\alpha|-d s>0$, one obtains bounds

$$
\left\|A^{\alpha} Q_{\lambda}\right\|_{p \rightarrow q} \leqslant a \lambda^{-(n-|\alpha|-d s) / m}
$$

for all $\lambda \geqslant \lambda_{0}$, where $Q_{\lambda}=\left(\lambda I+\Delta_{m}\right)^{-1 / 2}$, by the usual Laplace transform arguments.

It is also necessary to have precise estimates on the $n$-th derivatives of the square root of the resolvent of $\Delta_{m}$. It follows from [5] that one has bounds

$$
\left(\sum_{|\alpha|=n}\left\|A^{\alpha} Q_{\lambda_{0}} \varphi\right\|_{p}^{p}\right)^{1 / p} \leqslant c_{p}\left(\lambda_{0}\right)\|\varphi\|_{p}
$$

for all $\varphi \in L_{p}$ and all $p \in\langle 1, \infty\rangle$. Now we argue that one can improve these estimates uniformly in $\lambda$ if $p$ is sufficiently close to 2 . 
Lemma 2.1. For each $\varepsilon>0$ there exist $\delta \in\langle 0,1 / 2\rangle$ and $\lambda_{0}>0$ such that

$$
\left(\sum_{|\alpha|=n}\left\|A^{\alpha} Q_{\lambda} \varphi\right\|_{p}^{p}\right)^{1 / p} \leqslant(1+\varepsilon)\|\varphi\|_{p}
$$

uniformly for $\varphi \in L_{p}, \lambda \geqslant \lambda_{0}$ and $p \in\langle 1, \infty\rangle$ with $|1 / 2-1 / p| \leqslant \delta$.

Proof: First note that

$$
\left(\sum_{|\alpha|=n}\left\|A^{\alpha} Q_{\lambda} \varphi\right\|_{2}^{2}\right)^{1 / 2}=\left\|\Delta_{m}^{1 / 2}\left(\lambda I+\Delta_{m}\right)^{-1 / 2} \varphi\right\|_{2} \leqslant\|\varphi\|_{2}
$$

for all $\varphi \in L_{2}$ and all $\lambda>0$.

Secondly, if $\lambda_{0}$ is large enough then the semigroup generated by $\lambda_{0} I+\Delta_{m}$ on $L_{p}$ is exponentially decreasing and holomorphic in the right half-plane. Therefore, for all $p \in\langle 1, \infty\rangle$ the operator $\lambda_{0} I+\Delta_{m}$ has a bounded $H_{\infty}(\Lambda(\theta))$-functional calculus, in the sense of [6], for any $\theta \in\{0, \pi / 2\rangle$, by [9, Theorem 3.1]. So there exists $c_{p}>0$ such that

$$
\left\|f\left(\lambda_{0} I+\Delta_{m}\right)\right\|_{p \rightarrow p} \leqslant c_{p} \sup \{|f(z)|: z \in \Lambda(\theta)\}
$$

for all $f \in H_{\infty}(\Lambda(\theta))$. Hence application of this estimate to the holomorphic function $f(z)=z^{1 / 2}\left(\lambda-\lambda_{0}+z\right)^{-1 / 2}$ yields

$$
\left\|\left(\lambda_{0} I+\Delta_{m}\right)^{1 / 2}\left(\lambda I+\Delta_{m}\right)^{-1 / 2}\right\|_{p \rightarrow p}=\left\|f\left(\lambda_{0} I+\Delta_{m}\right)\right\|_{p \rightarrow p} \leqslant c_{p}
$$

for all $\lambda \geqslant \lambda_{0}$. One then concludes from combination of (7) and (9) that

$$
\left(\sum_{|\alpha|=n}\left\|A^{\alpha} Q_{\lambda} \varphi\right\|_{p}^{p}\right)^{1 / p} \leqslant c_{p}\left(\lambda_{0}\right)\left\|\left(\lambda_{0} I+\Delta_{m}\right)^{1 / 2}\left(\lambda I+\Delta_{m}\right)^{-1 / 2} \varphi\right\|_{p} \leqslant c_{p} c_{p}\left(\lambda_{0}\right)\|\varphi\|_{p}
$$

for all $p \in\langle 1, \infty)$ and $\varphi \in L_{p}$, uniformly for $\lambda \geqslant \lambda_{0}>0$.

Now let $Y=G \times\left\{1,2, \ldots, d^{n}\right\}$ with the natural sum measure, using left Haar measure on each copy of $G$. Then for all $\lambda \geqslant \lambda_{0}$ the operator $T_{\lambda}: L_{p}(G) \rightarrow L_{p}(Y)$ defined by $T_{\lambda} \varphi=\left(A^{\alpha_{1}} Q_{\lambda} \varphi, \ldots, A^{\alpha_{d^{n}}} Q_{\lambda} \varphi\right)$, where $\alpha_{i}$ is an enumeration of the $d^{n}$ multi-indices with $\left|\alpha_{i}\right|=n$, satisfies $\left\|T_{\lambda}\right\|_{L_{p}(G) \rightarrow L_{p}(Y)} \leqslant c_{p} c_{p}\left(\lambda_{0}\right)$ by the preceding estimate and $\left\|T_{\lambda}\right\|_{L_{2}(G) \rightarrow L_{2}(Y)} \leqslant 1$ by (8). Hence by interpolation for all $\varepsilon>0$ there exists $\delta \in\langle 0,1 / 2\rangle$ such that $\left\|T_{\lambda}\right\|_{L_{p}(G) \rightarrow L_{p}(Y)} \leqslant 1+\varepsilon$ uniformly for all $\lambda \geqslant \lambda_{0}$ and those $p$ such that $|1 / 2-1 / p| \leqslant \delta$. But this is equivalent to the statement of the lemma. 
The next lemma provides the crucial estimates on the resolvent of $H$ by expressing it as a perturbation of a multiple of $\Delta_{m}$ following a technique of [4, Chapter 1 , Section 4], which also occurs in [3, Proposition 3.1]. Let

$$
P=\sum_{|\alpha|=|\beta|=n}\left(A^{\alpha}\right)^{*} c_{\alpha, \beta} A^{\beta}
$$

denote the principal part of $H$.

LEMMA 2.2. There exist $\lambda_{1}>0, \delta \in\left\langle 0,(2 d)^{-1}\right\rangle$ and $a>0$ such that

$$
\left\|A^{\alpha}(\lambda I+P)^{-1} A^{\beta}\right\|_{p \rightarrow p} \leqslant a \lambda^{-(m-|\alpha|-|\beta|) / m}
$$

for all $\alpha, \beta$ with $|\alpha| \leqslant n$ and $|\beta| \leqslant n$ and

$$
\left\|A^{\alpha}(\lambda I+P)^{-1} A^{\beta}\right\|_{2 \rightarrow p} \leqslant a \lambda^{-(m-|\alpha|-|\beta|-d s) / m}
$$

for all $\alpha, \beta$ with $|\alpha| \leqslant n$ and $|\beta|<n$ uniformly for all $\lambda \geqslant \lambda_{1}$ and $p \in[2, \infty\rangle$ with $s=1 / 2-1 / p \leqslant \delta$.

PROOF: It suffices, by interpolation, to prove bounds with $a$ dependent on $p$.

Let $C=\left(c_{\alpha, \beta}\right)$ denote the $d^{n} \times d^{n}$-matrix formed by the coefficients with $|\alpha|=$ $n=|\beta|$ and set $\|C\|_{p}=\sup _{g \in G}\|C(g)\|_{p \rightarrow p}$, where $\|C(g)\|_{p \rightarrow p}$ denotes the $l_{p}$-norm of the matrix $C(g)$. Set $N=\mu_{C}^{-1}\|C\|_{2}^{2}, \sigma=\mu_{C}\|C\|_{2}^{-1}$ and $\tilde{C}=I-N^{-1} C$. Then

$$
|\tilde{C} \xi|^{2}=|\xi|^{2}-2 N^{-1} \operatorname{Re}(\xi, C \xi)+N^{-2}|C \xi|^{2} \leqslant\left(1-\sigma^{2}\right)|\xi|^{2}
$$

for all $\xi \in \mathbf{C}^{d^{n}}$. So $\|\widetilde{C}\|_{2}^{2} \leqslant 1-\sigma^{2}<1$. But $\|\widetilde{C}\|_{\infty} \leqslant d^{n}\|\tilde{C}\|_{2}$ and $\|\widetilde{C}\|_{1} \leqslant d^{n}\|\widetilde{C}\|_{2}$. Hence, by interpolation,

$$
\|\widetilde{C}\|_{p} \leqslant d^{n(1-2 / p)}\|\widetilde{C}\|_{2} \leqslant d^{n(1-2 / p)}\left(1-\sigma^{2}\right)^{1 / 2}=d^{n|1 / q-1 / p|}\left(1-\sigma^{2}\right)^{1 / 2}
$$

for all $p \in[2, \infty]$, with $q$ conjugate to $p$, and then, by a similar argument, for all $p \in[1,2]$. Therefore one may choose $\delta_{0} \in(0,1 / 2)$ such that

$$
\|\tilde{C}\|_{p} \leqslant 1-4^{-1} \sigma^{2}<1
$$

for all $p \in[1, \infty]$ satisfying $|1 / 2-1 / p| \leqslant \delta_{0}$.

Since $P=N\left(\Delta_{m}-\widetilde{P}\right)$ with $\tilde{P}=\sum_{|\alpha|=|\beta|=n}\left(A^{\alpha}\right)^{*} \widetilde{c}_{\alpha, \beta} A^{\beta}$ one has a formal representation

$$
N(\lambda N I+P)^{-1}=Q_{\lambda}\left(I-B_{\lambda}\right)^{-1} Q_{\lambda}
$$


where

$$
B_{\lambda}=Q_{\lambda} \tilde{P} Q_{\lambda}=\sum_{|\alpha|=|\beta|=n}\left(A^{\alpha} Q_{\lambda}\right)^{*} \tilde{c}_{\alpha, \beta}\left(A^{\beta} Q_{\lambda}\right)
$$

which we argue is well-defined on the $L_{p}$-spaces with $p$ sufficiently close to 2 .

Let $\varepsilon \in\langle 0,1]$ and fix $\delta$ such that the estimates of Lemma 2.1 are valid. Then for all $p \in\langle 1, \infty\rangle$ with $|1 / 2-1 / p| \leqslant \delta, \varphi \in L_{p} \cap L_{2}$ and $\chi \in L_{q} \cap L_{2}$, where $q$ is again conjugate to $p$, one has

$$
\begin{aligned}
\left|\left(\chi, B_{\lambda} \varphi\right)\right| & \leqslant\|\widetilde{C}\|_{p}\left(\sum_{|\alpha|=|\beta|=n}\left\|A^{\alpha} Q_{\lambda} \chi\right\|_{q}^{q}\right)^{1 / q}\left(\sum_{|\alpha|=|\beta|=n}\left\|A^{\beta} Q_{\lambda} \varphi\right\|_{p}^{p}\right)^{1 / p} \\
& \leqslant\left(1-4^{-1} \sigma^{2}\right)(1+\varepsilon)^{2}\|\chi\|_{q}\|\varphi\|_{p}
\end{aligned}
$$

uniformly for all $\lambda \geqslant \lambda_{0}$ whenever $|1 / q-1 / 2|=|1 / 2-1 / p| \leqslant \delta \wedge \delta_{0}$, where the last estimate uses (10). Hence by choosing $\varepsilon$ sufficiently small one may ensure that

$$
\left\|B_{\lambda}\right\|_{p \rightarrow p}<1-8^{-1} \sigma^{2}
$$

for all $p$ with $|1 / 2-1 / p| \leqslant \delta$, possibly by reducing the value of $\delta$. Therefore the $\left(I-B_{\lambda}\right)^{-1}$ are defined as bounded operators, by a norm convergent power series, on each of the $L_{p}$-spaces with $|1 / 2-1 / p| \leqslant \delta$ and their norms are uniformly bounded for $\lambda \geqslant \lambda_{0}$. Then, however, the representation (11) is well-defined for this range of $p$ and $\lambda$ and can be used to obtain the estimates of the lemma.

First, for all $|\alpha| \leqslant n,|\beta| \leqslant n$ and $p$ with $|1 / 2-1 / p| \leqslant \delta \wedge(1 / d)$ one has

$$
\begin{aligned}
\left\|A^{\alpha}(\lambda N I+P)^{-1} A^{\beta}\right\|_{p \rightarrow p} & \leqslant N^{-1}\left\|A^{\alpha} Q_{\lambda}\right\|_{p \rightarrow p}\left(1-\left\|B_{\lambda}\right\|_{p \rightarrow p}\right)^{-1}\left\|A^{\beta *} Q_{\lambda}\right\|_{q \rightarrow q} \\
& \leqslant N^{-1} \lambda^{-(n-|\alpha|) / m} 8 \sigma^{-2} \lambda^{-(n-|\beta|) / m}=a \lambda^{-(n-|\alpha|-|\beta|) / m},
\end{aligned}
$$

by (6) and (7), uniformly for $\lambda \geqslant \lambda_{0}$. Here $\beta_{*}$ denotes the multi-index obtained from $\beta$ by reversing its order. Finally,

$$
\begin{aligned}
\left\|A^{\alpha}(\lambda N I+P)^{-1} A^{\beta}\right\|_{2 \rightarrow p} & \leqslant N^{-1}\left\|A^{\alpha} Q_{\lambda}\right\|_{p \rightarrow p}\left(1-\left\|B_{\lambda}\right\|_{p \rightarrow p}\right)^{-1}\left\|A^{\beta^{*}} Q_{\lambda}\right\|_{q \rightarrow 2} \\
& \leqslant N^{-1} a \lambda^{-(n-|\alpha|) / m} 8 \sigma^{-2} a \lambda^{-(n-|\beta|-d(1 / q-1 / 2)) / m} \\
& =a \lambda^{-(m-|\alpha|-|\beta|-d s) / m}
\end{aligned}
$$

if $\delta$ is small enough. Thus the statements of the lemma are valid with $\lambda_{1}=N \lambda_{0}$.

Now we can extend (5) to the $\alpha$ with $|\alpha|=n$ for $p$ close to 2 . 
Lemma 2.3. Fix $\delta$ as in Lemma 2.2. Then there exist $a>0$ and $\omega \geqslant 0$ such that

$$
\left\|A^{\alpha} S_{t}\right\|_{2 \rightarrow p} \leqslant a t^{-(n+d s) / m} e^{\omega t}
$$

uniformly for all $\alpha$ with $|\alpha|=n, t>0$ and $p \in[2, \infty\rangle$ with $s=1 / 2-1 / p \leqslant \delta$.

Proof: It follows from the representation

$$
A^{\alpha} S_{t}=A^{\alpha}(\lambda I+P)^{-1}(\lambda I+H) S_{t}+A^{\alpha}(\lambda I+P)^{-1}(P-H) S_{t}
$$

that

$\left\|A^{\alpha} S_{t}\right\|_{2 \rightarrow p} \leqslant\left\|A^{\alpha}(\lambda I+P)^{-1}\right\|_{2 \rightarrow p}\left\|(\lambda I+H) S_{t}\right\|_{2 \rightarrow 2}+\left\|A^{\alpha}(\lambda I+P)^{-1}(P-H) S_{t}\right\|_{2 \rightarrow p}$.

There are two terms on the right hand side which we denote by $L(t)$ and $R(t)$, respectively. We set $\lambda=t^{-1}$. The leading term $L(t)$ can be bounded by the second estimate of Lemma 2.2, with $|\alpha|=n$ and $|\beta|=0$, together with (3). One finds

$$
L(t) \leqslant a \lambda^{-(n-d s) / m}\left(\lambda+t^{-1}\right) e^{\omega t} \leqslant a t^{-(n+d s) / m} e^{\omega t}
$$

first for $t \leqslant \lambda_{1}^{-1}$ and then, by the semigroup property, for all $t>0$ with possibly increased values of $a$ and $\omega$. Alternatively, the remainder $R(t)$ is bounded by

$$
\begin{aligned}
R(t) \leqslant \sum_{\beta, \gamma}^{\prime}\left\|A^{\alpha}(\lambda I+P)^{-1} A^{\beta}\right\|_{p \rightarrow p}\left\|c_{\beta, \gamma}\right\|_{\infty}\left\|A^{\gamma} S_{t}\right\|_{2 \rightarrow p} & \\
& +\sum_{\beta, \gamma}^{\prime \prime}\left\|A^{\alpha}(\lambda I+P)^{-1} A^{\beta}\right\|_{2 \rightarrow p}\left\|c_{\beta, \gamma}\right\|_{\infty}\left\|A^{\gamma} S_{t}\right\|_{2 \rightarrow 2}
\end{aligned}
$$

where the first sum is over the $\beta, \gamma$ with $|\beta|=n$ and $|\gamma| \leqslant n-1$ and the second over $|\beta| \leqslant n-1$ and $|\gamma| \leqslant n$. But these terms are bounded by the first estimate of Lemma 2.2 and (5), and the second estimate of Lemma 2.2 and (4), respectively. One finds

$$
R(t) \leqslant a t^{-(n-d s) / m} e^{\omega t}\left(\sum_{|\beta|+|\gamma|<m}\left\|c_{\beta, \gamma}\right\|_{\infty} t^{(m-|\beta|-|\gamma|) / m}\right)
$$

first for small $t$ and then, by increasing $a$ and $\omega$, for all $t>0$. Combination of these estimates gives the desired result and in fact the final estimate can be written in the form

$$
\left\|A^{\alpha} S_{t}\right\|_{2 \rightarrow p} \leqslant a t^{-(n-d s) / m} e^{\omega t}\left(\sum_{|\beta|+|\gamma| \leqslant m}\left\|c_{\beta, \gamma}\right\|_{\infty} t^{(m-|\beta|-|\gamma|) / m}\right) .
$$

This will be useful in the next step of the proof. 
REMARK 2.4. It is worth noting that the estimates of Lemma 2.3 combined with the usual perturbation expansion of the resolvent allow one to convert the bounds on the derivatives $A^{\alpha}(\lambda I+P)^{-1} A^{\beta}$ into analogous bounds on the derivatives $A^{\alpha}(\lambda I+H)^{-1} A^{\beta}$ at the cost of increasing the value of $\lambda_{1}$.

We next use the bounds of Lemma 2.3 together with the Sobolev inequalities

$$
\left\|A^{\alpha} \varphi\right\|_{\infty} \leqslant \varepsilon^{n-|\alpha|-d / p} \sup _{|\beta|=n}\left\|A^{\beta} \varphi\right\|_{p}+c_{p} \varepsilon^{-|\alpha|-d / p}\|\varphi\|_{p}
$$

which are valid for all $\varepsilon \in\langle 0,1]$ whenever $n-|\alpha|-d / p>0$, in order to obtain bounds from $L_{2}$ into $L_{\infty}$. Setting $\varepsilon=t^{1 / m}$ and $1 / p=1 / 2-\delta$, with $\delta$ as in Lemmas 2.2 and 2.3 gives

$$
\left\|A^{\alpha} S_{t}\right\|_{2 \rightarrow \infty} \leqslant t^{(n-|\alpha|-d / p) / m} \sup _{|\beta|=n}\left\|A^{\beta} S_{t}\right\|_{2 \rightarrow p}+c_{p} t^{-(|\alpha|+d / p) / m}\left\|S_{t}\right\|_{2 \rightarrow p}
$$

for $t \in\langle 0,1]$ and $|\alpha|<2^{-1}(m-d)+d \delta$. Note that $2^{-1}(m-d)+d \delta$ is not an integer. But then the estimates of Lemma 2.3 together with (5) give bounds

$$
\left\|A^{\alpha} S_{t}\right\|_{2 \rightarrow \infty} \leqslant a t^{-(d / 2+|\alpha|) / m} e^{\omega t}
$$

for $t \in\langle 0,1]$ and then, by enlarging $a$ and $\omega$, for all $t>0$. Since similar bounds are valid for the adjoint semigroup one concludes that

$$
\left\|A^{\alpha} S_{t} A^{\beta *}\right\|_{1 \rightarrow \infty} \leqslant\left\|A^{\beta} S_{t / 2}^{*}\right\|_{2 \rightarrow \infty}\left\|A^{\alpha} S_{t / 2}\right\|_{2 \rightarrow \infty} \leqslant a t^{-d / m} t^{-(|\alpha|+|\beta|) / m} e^{\omega t}
$$

for all $t>0$ and $\alpha, \beta$ with $|\alpha|<2^{-1}(m-d)+d \delta$ and $|\beta|<(m-d) / 2+d \delta$. Then by the Dunford-Pettis theorem [1, Theorem 1.3] the operator $A^{\alpha} S_{t} A^{\beta *}$ has a kernel $K_{t}^{(\alpha, \beta)} \in L_{\infty}(G \times G)$ satisfying $\left\|K_{t}^{(\alpha, \beta)}\right\|_{\infty} \leqslant a t^{-d / m} t^{-(|\alpha|+|\beta|) / m} e^{\omega t}$. Set $K_{t}=K_{t}^{(\alpha, \beta)}$ if $|\alpha|=|\beta|=0$.

Next, the Hölder space $C^{\tau}$ is defined for $\tau=N+\sigma$ with $N \in \mathbf{N}_{0}$ and $\sigma \in\langle 0,1\rangle$ as the subspace of $L_{\infty ; N}$ for which the norm

$$
\|\varphi\|_{C^{\tau}}=\sup _{0<|k| \leqslant 1} \sup _{|\alpha| \leqslant N}|k|^{-\sigma}\left\|(I-L(k)) A^{\alpha} \varphi\right\|_{\infty}
$$

is finite. Then one has the Sobolev inequalities

$$
\|\varphi\|_{C^{\tau}} \leqslant \varepsilon^{n-\tau-d / p} \sup _{|\alpha|=n}\left\|A^{\alpha} \varphi\right\|_{p}+c_{p} \varepsilon^{-d / p}\|\varphi\|_{p}
$$

which are valid for all $\varepsilon \in\langle 0,1]$ whenever $n-\tau>d / p$. In particular if $1 / p=1 / 2-\delta$ these inequalities are valid for all $\tau \in\left\langle 0,2^{-1}(m-d)+d \delta\right\rangle \backslash N$. Now the arguments in the 
above proof can be repeated and one deduces that for all $\tau=|\alpha|+\sigma<2^{-1}(m-d)+d \delta$ there are $a$ and $\omega$ such that

$$
|k|^{-\sigma}\left\|(I-L(k)) A^{\alpha} S_{t}\right\|_{2 \rightarrow \infty} \leqslant\left\|S_{t}\right\|_{L_{2} \rightarrow C^{\tau}} \leqslant a t^{-(d / 2+\tau) / m} e^{\omega t}
$$

for all $t>0$ first for all $|k| \in\langle 0,1]$ and then, by the bounds (14), for all $k \in G$. Similar bounds are valid on the adjoint semigroup. Therefore combination with (14) gives

$$
\begin{aligned}
\left\|L(k) A^{\alpha} S_{t} A^{\beta_{*}} L\left(l^{-1}\right)-A^{\alpha} S_{t} A^{\beta_{*}}\right\|_{1 \rightarrow \infty} \\
\quad \leq a\left(|k|^{\sigma}|l|^{\sigma} t^{-2 \sigma / m}+|k|^{\sigma} t^{-\sigma / m}+|l|^{\sigma} t^{-\sigma / m}\right) t^{-d / m} t^{-(|\alpha|+|\beta|) / m} e^{\omega t}
\end{aligned}
$$

for all $\alpha, \beta$ and $\sigma \in\langle 0,1\rangle$ with $|\alpha|+\sigma<2^{-1}(m-d)+d \delta$ and $|\beta|+\sigma<2^{-1}(m-d)+d \delta$ and for all $t>0, k, l \in G$. So

$$
\begin{aligned}
\|(I-\tilde{L}(k, l)) & K_{t}^{(\alpha, \beta)} \|_{\infty} \\
& \leqslant a\left(|k|^{\sigma}|l|^{\sigma} t^{-2 \sigma / m}+|k|^{\sigma} t^{-\sigma / m}+|l|^{\sigma} t^{-\sigma / m}\right) t^{-d / m} t^{-(|\alpha|+|\beta|) / m} e^{\omega t}
\end{aligned}
$$

where $\widetilde{L}$ denotes the left translations on $G \times G$. Hence the measurable function $K_{t}^{(\alpha, \beta)}$ is continuous and the lemma of Du Bois-Reymond implies that $K_{t}$ is $|\alpha|$-times differentiable in the first variable and the derivatives are $|\beta|$-times differentiable in the second variable.

STEP 2. (Gaussian bounds) The Gaussian bounds on the kernel now follow by repetition of the foregoing arguments for the perturbed semigroup

$$
S_{t}^{\rho}=U_{\rho} S_{t} U_{\rho}^{-1}=e^{-t H_{\rho}}
$$

where $\rho \mapsto U_{\rho}$ is the unitary group of multiplication operators defined by $U_{\rho} \varphi=$ $e^{-\rho \psi} \varphi$, with real-valued $\psi \in C_{c}^{\infty}(G)$ and $H_{\rho}$ is the operator obtained from $H$ by the replacement $A_{i}$ by $A_{i}+\rho\left(A_{i} \psi\right)$. All subsequent bounds depend on derivatives $A^{\alpha} \psi$ of $\psi$ and are uniform over the set $D_{n}$ of real-valued $\psi \in C_{c}^{\infty}(G)$ with $\left\|A^{\alpha} \psi\right\|_{\infty} \leqslant 1$ for all $\alpha$ with $1 \leqslant|\alpha| \leqslant n$. First the bounds (3) are replaced by

$$
\left\|S_{t}^{\rho}\right\|_{2 \rightarrow 2} \leqslant e^{\omega\left(1+\rho^{m}\right) t}, \quad\left\|H_{\rho} S_{t}^{\rho}\right\|_{2 \rightarrow 2} \leqslant a t^{-1} e^{\omega\left(1+\rho^{m}\right) t}
$$

with $a$ and $\omega$ independent of $\rho$ and $\psi \in D_{n}$ (see [17, Lemma III.4.4]). Then

$$
\sup _{|\alpha|=n}\left\|A^{\alpha} S_{t}^{\rho} \varphi\right\|_{2}^{2} \leqslant \mu_{C}^{-1} \operatorname{Re}\left(S_{t}^{\rho} \varphi, P S_{t}^{\rho} \varphi\right)
$$

by ellipticity. But

$$
\operatorname{Re}(\chi, P \chi) \leqslant 2\left|\left(\chi, H_{\rho} \chi\right)\right|+a\left(1+\rho^{m}\right)\|\chi\|_{2}^{2}
$$


as a form bound by a calculation similar to the proof [17, Lemma II.4.5]. Thus

$$
\sup _{|\alpha|=n}\left\|A^{\alpha} S_{t}^{\rho}\right\|_{2 \rightarrow 2}^{2} \leqslant 2 \mu_{C}^{-1}\left\|S_{t}^{\rho}\right\|_{2 \rightarrow 2}\left\|H_{\rho} S_{t}^{\rho}\right\|_{2 \rightarrow 2}+a\left(1+\rho^{m}\right)\left\|S_{t}^{\rho}\right\|_{2 \rightarrow 2}^{2} \leqslant a t^{-1} e^{\omega\left(1+\rho^{m}\right) t}
$$

Then, by a standard $\varepsilon, \varepsilon^{-1}$ argument one obtains the analogue

$$
\left\|A^{\alpha} S_{t}^{\rho}\right\|_{2 \rightarrow 2} \leqslant a t^{-|\alpha| / m} e^{\omega\left(1+\rho^{m}\right) t}
$$

of (4) for all $\alpha$ with $|\alpha| \leqslant n$ and all $t, \rho$ and $\psi$. The arguments leading from (4) to (5) applied to (15) now yield

$$
\left\|A^{\alpha} S_{t}^{\rho}\right\|_{2 \rightarrow p} \leqslant a t^{-d s / m} t^{-|\alpha| / m} e^{\omega\left(1+\rho^{m}\right) t}
$$

for all $t>0, \rho \in \mathbf{R}, \psi \in D_{n}$ and all $\alpha$ with $|\alpha|<n$ and all $p \in[2, \infty)$ with $s=1 / 2-1 / p<1 / d$. But $P$ is the principal part of $H_{\rho}$ and

$$
P-H_{\rho}=\sum_{|\beta|+|\gamma|<m} A^{\beta} c_{\beta, \gamma}(\rho) A^{\gamma}
$$

where $\rho \mapsto c_{\beta, \gamma}(\rho)$ is a polynomial of order at most $m-|\beta|-|\gamma|$ and the coefficients are uniformly bounded for $\psi \in D_{n}$. Moreover, the $c_{\beta, \gamma}(\rho)$ are linear in the $c_{\beta, \gamma}$. Therefore the reasoning of Lemmas $2.1,2.2$ and 2.3 can be applied to $H_{\rho}$ and $S_{t}^{\rho}$ to obtain the bounds

$$
\begin{aligned}
& \left\|A^{\alpha} S_{t}^{\rho}\right\|_{2 \rightarrow p} \\
& \quad \leqslant a t^{-(n+d s) / m} e^{\omega\left(1+\rho^{m}\right) t}\left(\sum_{|\beta|+|\gamma| \leqslant m}\left\|c_{\beta, \gamma}\right\|_{\infty}\left(1+\rho^{(m-|\beta|-|\gamma|)}\right) t^{(m-|\beta|-|\gamma|) / m}\right)
\end{aligned}
$$

for all $\alpha$ with $|\alpha|=n$ in place of (13). But by increasing the values of $a$ and $\omega$ one has

$$
\left\|A^{\alpha} S_{t}^{\rho}\right\|_{2 \rightarrow p} \leqslant a t^{-(n+d s) / m} e^{\omega\left(1+\rho^{m}\right) t}
$$

for all $t>0$ uniformly for $\rho \in \mathbf{R}, \psi \in D_{n}$ and $p \in[2, \infty\rangle$ with $s=1 / 2-1 / p \leqslant \delta$.

At this point one can repeat the arguments following the proof of Lemma 2.3 with $S_{t}$ replaced by $S_{t}^{\rho}$ and using (15), (16) and (17) in place of (4), (5) and (12) to conclude that

$$
\left\|A^{\alpha} S_{t}^{\rho} A^{\beta *}\right\|_{1 \rightarrow \infty} \leqslant a t^{-d / m} t^{-(|\alpha|+|\beta|) / m} e^{\omega\left(1+\rho^{m}\right) t}
$$

for all $t>0, \rho \in \mathbf{R}, \psi \in D_{n}$ and $\alpha, \beta$ with $|\alpha|<2^{-1}(m-d)+d \delta$ and $|\beta|<$ $2^{-1}(m-d)+d \delta$. The $a$ and $\omega$ are independent of $\rho, \psi$ and $t$. Then

$$
\left\|U_{\rho} A^{\alpha} S_{t} A^{\beta *} U_{\rho}^{-1}\right\|_{1 \rightarrow \infty} \leqslant a t^{-d / m} t^{-(|\alpha|+|\beta|) / m} e^{\omega\left(1+\rho^{m}\right) t} .
$$


As the kernel of $U_{\rho} A^{\alpha} S_{t} A^{\beta *} U_{\rho}^{-1}$ is given by $(g, h) \mapsto(-1)^{|\beta|} e^{\rho(\psi(g)-\psi(h))}\left(A^{\alpha} B^{\beta} K_{t}\right)(g ; h)$ it then follows that

$$
\left|\left(A^{\alpha} B^{\beta} K_{t}\right)(g ; h)\right| \leqslant a t^{-d / m} t^{-(|\alpha|+|\beta|) / m} e^{\omega t} e^{\omega \rho^{m} t-|\rho||\psi(g)-\psi(h)|}
$$

and the Gaussian bounds (2) follow by minimising over $\rho \in \mathbf{R}$ and $\psi \in D_{n}$. Note that the distance which naturally enters these estimates is given by

$$
d_{n}(g ; h)=\sup _{\psi \in D_{n}}|\psi(g)-\psi(h)|
$$

But this distance is equivalent to the normal distance $(g, h) \mapsto\left|g h^{-1}\right|$ (see $[17$, pp.201202]).

Finally, similar estimates can be deduced for the Hölder continuity of the derivatives of the kernel. Specifically, for all $\alpha, \beta$ and $\sigma \in\langle 0,1\rangle$ with $|\alpha|+\sigma<2^{-1}(m-d)+d \delta$ and $|\beta|+\sigma<2^{-1}(m-d)+d \delta$ one obtains

$$
\begin{aligned}
& \left|e^{-\rho\left(\psi\left(k^{-1} g\right)-\psi\left(l^{-1} h\right)\right)}\left(A^{\alpha} B^{\beta} K_{t}\right)\left(k^{-1} g ; l^{-1} h\right)-e^{-\rho(\psi(g)-\psi(h))}\left(A^{\alpha} B^{\beta} K_{t}\right)(g ; h)\right| \\
& \quad \leqslant a\left(|k|^{\sigma}|l|^{\sigma} t^{-2 \sigma / m}+|k|^{\sigma} t^{-\sigma / m}+|l|^{\sigma} t^{-\sigma / m}\right) t^{-d / m} t^{-(|\alpha|+|\beta|) / m} e^{\omega\left(1+\rho^{m}\right) t}
\end{aligned}
$$

uniformly for all $g, h, k, l \in G, t>0, \rho \in \mathbf{R}$ and $\psi \in D_{n}$. Using (2) one can minimise the above bounds and obtain the second bounds of Theorem 1.1 by a slightly more complicated argument which we omit (see [11, proof of Proposition 4.5]).

STEP 3. (Non-unimodular $G$ ) If $G$ is non-unimodular it is necessary to analyse the left differential operator $H$ on the spaces $L_{\widehat{p}}=L_{p}(G ; d \widehat{g})$ formed with respect to right Haar measure. In fact the kernel $K_{t}$ is bounded if and only if the semigroup $S_{t}$ extends to a bounded operator from $L_{\hat{1}}$ to $L_{\infty}$.

The left derivatives $A_{i}$ are no longer skew-adjoint on $L_{\widehat{2}}$ but the $L_{\widehat{2}}$ adjoint is given by $\widehat{A_{i}^{*}}=-A_{i}+b_{i} I$ where $b_{i}=\left(A_{i} \Delta\right)(e)$ is the derivative of the modular function $\Delta$ at the identity $e$. Nevertheless $H$ is still defined on $L_{\widehat{2}}$ as a maximal accretive operator associated with a sectorial form

$$
\widehat{h}(\varphi, \psi)=\sum_{|\alpha|,|\beta| \leqslant n}\left\langle A^{\alpha} \varphi, \widehat{c}_{\alpha, \beta} A^{\beta} \psi\right\rangle
$$

where $\langle\cdot, \cdot\rangle$ denotes the scalar product on $L_{\widehat{2}}$ and the $\widehat{c}_{\alpha, \beta}$ are linear in the $c_{\alpha, \beta}$ and polynomial in the $b_{i}$, but the principal coefficients are unchanged. Hence $H$ generates a semigroup $\widehat{S}$ on $L_{\widehat{2}}$ with kernel $\widehat{K}$ and now one has

$$
\sup _{g, h \in G}\left|\widehat{K}_{t}(g ; h)\right|=\left\|\widehat{S}_{t}\right\|_{\hat{1} \rightarrow \infty}
$$


et cetera. Once one proves that $\widehat{K}$ satisfies Gaussian bounds it is then straightforward to verify that $\widehat{S}_{t}\left(L_{\widehat{2}} \cap L_{2}\right) \subseteq\left(L_{\widehat{2}} \cap L_{2}\right)$, its extension to $L_{2}$ is generated by the $L_{2}$ version of $H$ and $\widehat{K}_{t}=K_{t}$. Therefore one must now repeat the proofs of Steps 1 and 2 but relative to the $L_{\widehat{p}}$ spaces. This presents no difficulty.

First, the Sobolev embeddings inequalities used in the proofs remain valid for left derivatives on the $L_{\widehat{p}}$ spaces (see [17, Appendix B.2]) and, in fact, they fail for the $L_{p}$-spaces. Secondly, the principal part $P$ is now defined relative to $L_{\hat{2}}$ as the operator associated with the form

$$
p(\varphi, \psi)=\sum_{|\alpha|,|\beta|=n}\left\langle A^{\alpha} \varphi, c_{\alpha, \beta} A^{\beta} \psi\right\rangle
$$

and the approximant $\Delta_{m}$ is introduced by the form $\varphi \mapsto \sum_{|\alpha|=n}\left\|A^{\alpha} \varphi\right\|_{\widehat{2}}^{2}$. After these changes the proofs proceed as previously. We omit further details.

\section{REFERENCES}

[1] W. Arendt and A.V. Bukhvalov, 'Integral representations of resolvents and semigroups', Forum Math. 6 (1994), 111-135.

[2] P. Auscher, T. Coulhon and P. Tchamitchian, 'Absence de principe du maximum pour certaines équations paraboliques complexes', Colloq. Math. 71 (1996), 87-95.

[3] P. Auscher, A. McIntosh and P. Tchamitchian, Heat kernels of second order complex elliptic operators and their applications, Research Report 94-164 (Maquarie University, Sydney, Australia, 1994.).

[4] A. Bensoussan, J.L. Lions and G. Papanicolaou, Asymptotic analysis for periodic structures, Studies in Mathematics and its Applications 5 (North-Holland, Amsterdam, New York, Oxford, Tokyo, 1978).

[5] R.J. Burns, A.F.M. ter Elst and D.W. Robinson, ' $L_{p}$-regularity of subelliptic operators on Lie groups', J. Operator Theory 31 (1994), 165-187.

[6] M. Cowling, I. Doust, A. McIntosh and A. Yagi, 'Banach space operators with a bounded $H^{\infty}$ functional calculus', J. Austral. Math. Soc. Ser. A 60 (1996), 51-89.

[7] E.B. Davies, 'Uniformly elliptic operators with measurable coefficients', J. Funct. Anal. 132 (1995), 141-169.

[8] E.B. Davies, 'Limits on $L_{p}$ regularity of self-adjoint elliptic operators.', J. Differential Equations (to appear).

[9] A.F.M. ter Elst and D.W. Robinson, 'Functional analysis of subelliptic operators on Lie groups', J. Operator Theory 31 (1994), 277-301.

[10] A.F.M. ter Elst and D.W. Robinson, 'Elliptic operators on Lie groups', Acta Appl. Math. 44 (1996), 133-150.

[11] A.F.M. ter Elst and D.W. Robinson, Second-order subelliptic operators on Lie groups I: complex uniformly continuous principal coefficients, Research Report MRR 035-96 (The Australian National University, Canberra, Australia, 1996). 
[12] M. Giaquinta, Multiple integrals in the calculus of variations and nonlinear elliptic systems, Annals of Mathematics Studies 105 (Princeton University Press, Princeton, 1983).

[13] E.D. Giorgi, 'Sulla differenziabilità e l'analiticità delle estremali degli integrali multipli regolari', Mem. Accad. Sci. Torino Cl. Sci. Fis. Mat. Natur. 3 (1957), 25-43.

[14] T. Kato, Perturbation theory for linear operators, Grundlehren der mathematischen Wissenschaften 132, (second edition) (Springer-Verlag, Berlin, Heidelberg, New York, 1984).

[15] C.B. Morrey, Multiple integrals in the calculus of variations (Springer-Verlag, Berlin, Heidelberg, New York, 1966).

[16] J. Nash, 'Continuity of solutions of parabolic and elliptic equations', Amer. J. Math. 80 (1958), 931-954.

[17] D.W. Robinson, Elliptic operators and Lie groups, Oxford Mathematical Monographs (Oxford University Press, Oxford, 1991).

\section{Department of Mathematics and Computing Science}

Eindhoven University of Technology

PO Box 513

5600 MB Eindhoven

The Netherlands
Centre for Mathematics and its Applications School of Mathematical Sciences

Australian National University

Canberra ACT 0200

Australia 